\title{
ON AN OPEN QUESTION CONCERNING FIXED POINTS
}

WAYMAN L. STROTHER ${ }^{1}$

A space $X$ is said to have the f.p.p. (fixed point property) if every continuous function $f$ from $X$ to $X$ has a fixed point. Whether if $X$ and $Y$ have the f.p.p. then $X \times Y$ has the f.p.p. is an open question.

A space $X$ is said to have the F.p.p. (fixed point property for multivalued functions) if every continuous multi-valued function $F$ from $X$ to $X$ has a fixed point, i.e., a point $x$ such that $x \in F(x)$. Interest in fixed points for multi-valued functions leads one to question under what conditions on the spaces $X$ and $Y$ and on the multi-valued function $F$ on $X$ to $Y$ there will exist a continuous trace $f$ of $F$, that is, a continuous function $f$ on $X$ to $Y$ such that $f(x) \in F(x)$ for all $x$. For some specific multi-valued functions $F$ it is possible to produce a continuous trace. It is by use of these traces that most of the fixed point theorems in the literature for multi-valued functions are proved. In fact the open question mentioned above (which is concerned only with single-valued functions) can be answered if one can produce continuous traces of two particular multi-valued functions. This paper proves some fixed point theorems by producing continuous traces, shows that a continuous multi-valued function need not have a continuous trace, and gives an example which indicates that a general theorem on the existence of a continuous trace is not likely to be established without strong conditions on $F$ regardless of what conditions are placed on $X$ and $Y$. This example answers in the negative the generalization of the above open question to the multi-valued case, exhibits a continuous multi-valued function which has no continuous trace, shows that the general Tychonoff cube does not have the F.p.p., and shows that a space with the f.p.p. need not have the F.p.p.

Notation. By $\left\{x_{a}\right\} \rightarrow x_{0}$ we denote a sequence of points indexed by a directed set $A$ and converging to $x_{0}$. The directing relation in $A$ will be denoted by *.

Definition 1. Continuous. A multi-valued function on a space $X$ to a space $Y$ is said to be continuous at $x_{0}$ if $\left\{x_{a}\right\} \rightarrow x_{0}$ implies that $F\left(x_{0}\right)=$ cofinal limit $\left\{F\left(x_{a}\right)\right\}=$ residual limit $\left\{F\left(x_{a}\right)\right\} . F$ is said to be

Presented to the Society, November 23, 1951; received by the editors January 12 , 1953 and, in revised form, April 27, 1953.

1 This work was done under contract N7-onr-434, Task Order III, Navy Department Office of Naval Research. The author wishes to express his appreciation for the suggestions of Professor A. D. Wallace. 
continuous if it is continuous at every $x$ in $X$.

For the details of how this definition of continuity is related to the definitions used elsewhere in the literature see [1]. This definition is strong enough, however, to insure that the example is valid for functions continuous under the definitions used by Ratner [2], Wallace [3], Eilenberg and Montgomery [4], Kakutani [5], Banach and Mazur [6], and Michael [7].

Directly from the definition of trace we establish the following

LEMMA 1. Let $f$ be a trace of a multi-valued function $F$ on $X$ to $Y$ and let $x$ be a fixed point of $f$. Then $x$ is a fixed point of $F$.

From Lemma 1 it is clear that a sufficient condition for a continuous multi-valued function $F$ to have a fixed point is that $F$ have a continuous trace which has a fixed point. But this is not a necessary condition. In fact one obtains a continuous multi-valued function with a fixed point and no continuous trace by defining $F$ from the unit circle at the origin in the complex plane to itself by $F(z)=$ the two square roots of $z$.

\section{The example.}

Theorem 1. A bounded closed interval I of real numbers has the F.p.p.

Proof. Brouwer's theorem assures us that $I$ has the f.p.p. Hence in view of Lemma 1 it is sufficient to prove that every continuous function $\dot{F}$ on $I$ to $I$ has a continuous trace. We shall in fact prove that if $R$ is a bounded closed interval of real numbers and $F$ is a continuous multi-valued function on a space $X$ to $R$, then $F$ has a continuous trace $f$. Define $f$ on $X$ to $R$ by $f(x)=\operatorname{lub}\{y \mid y \in F(x)\}$.

It is known [1] that a multi-valued function from a space $X$ to a compact Hausdorff space $Y$ is continuous if and only if $x_{0} \in X$ implies:

(1) $F\left(x_{0}\right)$ is closed,

(2) $V$ open containing $F\left(x_{0}\right)$ implies that there exists an open set $U^{\prime}$ containing $x_{0}$ and such that whenever $x \in U^{\prime}$ then $F(x) \subset V$, and

(3) $y_{0} \in F\left(x_{0}\right), y_{0} \in V$, and $V$ open imply that there is an open set $U^{\prime \prime}$ containing $x_{0}$ such that whenever $x \in U^{\prime \prime}$ then $F(x) \cap V \neq 0$.

Let $V_{2 \phi}$ be an open interval of length $2 \phi$ with center $f\left(x^{\prime}\right)$, where $\phi$ is a positive real number. Then $V_{\phi}$ is also an open set containing $f\left(x^{\prime}\right)$. By (3) there is an open set $U^{\prime \prime}$ containing $x_{0}$ such that $x \in U^{\prime \prime}$ implies that $F(x) \cap V_{\phi} \neq 0$. Hence $x \in U^{\prime \prime}$ implies that lub $\{y \mid y \in F(x)\}$ $=f(x) \geqq f\left(x^{\prime}\right)-\phi$.

Let $V=\left\{y \mid y<\phi+f\left(x^{\prime}\right)\right\}$. This set $V$ is open containing $F\left(x^{\prime}\right)$. Hence (2) implies that there exists an open set $U^{\prime}$ containing $x^{\prime}$ such 
that $x \in U^{\prime}$ implies that $F(x) \subset V$. Then $x \in U^{\prime}$ implies $f(x)$ $=$ lub $\{y \mid y \in F(x)\} \leqq f\left(x^{\prime}\right)+\phi$. Let $U=U^{\prime} \cap U^{\prime \prime}$. Then $x \in U$ implies that $\left|f(x)-f\left(x^{\prime}\right)\right| \leqq \phi$, therefore $f(x) \in V_{2 \phi}$, and $f$ is continuous at $x^{\prime}$.

Theorem 1 established that $I$ has the F.p.p. Let $r$ be a continuous function which retracts the unit square $I \times I$ onto the unit disc $X$. Define $F$ from $X$ to $X$ as follows. If $x$ is the origin, let $F(x)=S$, where $S$ denotes the unit circle with center at the origin. If $x$ is not the origin: (a) Extend the segment from the origin through $x$ until it meets $S$ in a point $A$. (b) Draw a perpendicular at $x$ to the radius constructed in (a) and denote its intersections with $S$ as $B$ and $C$. (c) Consider the closed arc $B A C$ on $S$. Let $M B A C N$ be the closed arc of $S$ with center $A$, length twice the length of the arc $B A C$, and having end points $M$ and $N$. (d) Let $F(x)=M B A C N$. That $F$ has the three properties utilized in the proof of Theorem 1 is geometrically evident and hence $F$ is continuous. Define $G$ to be $i F r$ followed by a rotation of ninety degrees, where $i$ denotes the injection of $X$ into $I \times I$. The continuity of $G$ follows from [1, Proposition 18]. Now $x \in(I \times I)-S$ implies that $x \notin G(x)$ because $G(I \times I) \subset S$. Also $x \in S$ implies that $F(x)=x$ and the rotation moves $x$. Hence $G$ has no fixed point and consequently $I \times I$ does not have the F.p.p.

Cartesian and apex functions. Most theorems on fixed points for multi-valued functions demand either that $F(x)$ be a connected set for every $x$ or that $F(x)$ be a convex set for every $x$. The literature appears to be void of fixed point theorems with no condition on the image of a point. Theorem 1 above is such a theorem. The example above shows that the two-dimensional cube does not have the F.p.p. A simple extension of this result shows that no Tychonoff cube of dimension greater than one has the F.p.p. Then in order to prove theorems concerning fixed points for functions on a Tychonoff cube one must place some further conditions on $F$. The following theorems indicate that $F$ may enjoy much greater pointwise freedom than is allowed under the usual assumption that $F(x)$ is either convex or connected.

Definition 2. Cartesian function. Let $T=P I_{a}$ be a Tychonoff cube. A subset $Y_{0}$ of $T$ is called a $T$-cartesian subset if $Y_{0}=P M_{a}$, where $M_{a}$ is a subset of $I_{a}$. A function $F$ from a space $X$ to a space $Y$ is called a cartesian function if there exists a homeomorphism $h$ of $Y$ into some Tychonoff cube $T$ such that (1) $h(Y)$ is a retract of $T$ and (2) $x \in X$ implies that $h F(x)$ is a $T$-cartesian subset of $T$.

Theorem 2. Every continuous Cartesian function from a space $X$ to itself has a fixed point. 
Proof. Let $F$ be a continuous cartesian function on $X$ to itself. There is a Tychonoff cube $T=P I_{a}$, a homeomorphism $h$ of $X$ into $T$, and a retraction $r$ of $T$ onto $h(T)$ such that $x \in X$ implies that $h F(x)$ is a $T$-cartesian subset of $T$. Let $G(x)=i h F(x)$, where $i$ denotes the injection of $h(X)$ into $T$. Define $H$ on $T$ to $T$ by $H(x)=G h^{-1} r(x)$ $=i h F h^{-1} r(x)$ and define $H_{a}$ from $T$ to $I_{a}$ by $H_{a}(x)=$ the projection of $H(x)$ in $I_{a}$. That $H$ and $H_{a}$ are continuous is established in [1]. In the proof of Theorem 1 it was shown that $H_{a}$ has a continuous trace $f_{a}$. Kakutani [5] showed that if each $f_{a}$ is a continuous single-valued function, so is $f$ defined by $f(x)=P f_{a}(x)$. For each $a, f_{a}(x)$ is an element of $H_{a}(x)$ and $H(x)=P M_{a}(x)$, where $M_{a}$ is a subset of $I_{a}$, so that $f(x)$ $\in H(x)$. Now $f$ is a continuous single-valued function from a Tychonoff cube to itself and hence has a fixed point.

Let $x^{\prime}$ be a fixed point of $H$. Then $x^{\prime} \in H\left(x^{\prime}\right)=i h F h^{-1} r\left(x^{\prime}\right)$ $=h F h^{-1} r\left(x^{\prime}\right)$. Since $x^{\prime} \in T, r\left(x^{\prime}\right) \in h(T)$ and hence there exists $x^{\prime \prime} \in T$ such that $r\left(x^{\prime}\right)=h\left(x^{\prime \prime}\right)$. Then $x^{\prime} \in H\left(x^{\prime}\right)=h F h^{-1} h\left(x^{\prime \prime}\right)=h F\left(x^{\prime \prime}\right)$ $\subset h F(X) \subset h(X)$. The function $r$ retracts $T$ onto $h(X)$, hence $x^{\prime}=r\left(x^{\prime}\right)$ $=h\left(x^{\prime \prime}\right), x^{\prime \prime}=h^{-1}\left(x^{\prime}\right), x^{\prime} \in h F\left(x^{\prime \prime}\right)$, and $x^{\prime \prime}=h^{-1}\left(x^{\prime}\right) \in F\left(x^{\prime \prime}\right)$.

Definition 3. Apex set. Let $B$ be a closed subset of a Tychonoff cube $T=P I_{a}$. Denote by $B_{a}$ and $b_{a}$ the projections of $B$ and of $b$, respectively, in $I_{a}$. For a fixed $a$ denote lub $\left\{b_{a} \mid b_{a} \in B_{a}\right\}$ by $m\left(B_{a}\right)$. The set $B_{a}$ is closed and hence $m\left(B_{a}\right) \in B_{a}$. If there is only one point in $B$ which projects onto $m\left(B_{a}\right)$ we say that $B$ is an apex subset of $T$ with respect to $a$.

Definition 4. Apex function. A function $F$ from a space $X$ to a space $Y$ is called an apex function if there is a homeomorphism $h$ of $Y$ onto a retract of a Tychonoff cube $T=P I_{a}$ such that, for some fixed $a=a(1), x \in X$ implies that $h F(x)$ is an apex subset of $T$ with respect to $a(1)$.

Theorem 3. Every continuous apex function from a space $X$ to itself has a fixed point.

Proof. Let $G$ be a continuous apex function from $X$ to $X$. Then there is a Tychonoff cube $T=P I_{a}$, a homeomorphism $h$ of $X$ into $T$, a retraction $r$ of $T$ onto $h(X)$, and a fixed $a=a(1)$ such that $x \in X$ implies that $h G(x)$ is an apex subset of $T$ with respect to $a(1)$. The function $F=h G h^{-1} r$ is defined on $T$ to $T$. As in the proof of Theorem 2, $F_{a(1)}$ is continuous. By (1) in the proof of Theorem $1, t \in T$ implies that $F_{a(1)}(t)$ is closed and hence $t \in T$ implies that $m\left[F_{a(1)}(t)\right]$ is an element of $F_{a(1)}(t)$. The hypothesis that $G$ is an apex function implies that there is exactly one $t^{\prime} \in F(t)$ such that $t^{\prime}$ projects onto $m\left[F_{a(1)}(t)\right]$. Define a single-valued function $f$ from $T$ to $T$ by $f(t)=t^{\prime}$. 
Clearly $f$ is a trace of $F$.

Let $x^{0}$ be an element of $T$ and let $y^{0}$ be an element of $F\left(x^{0}\right)$. Let $\phi$ be a real number greater than zero and let $V_{a(1)}(\phi)$ contain $f_{a(1)}\left(x^{0}\right)$ $=y_{a(1)}^{0}$. By (3) in the proof of Theorem 1 there is an open set $U^{\prime \prime}$ containing $x^{0}$ such that whenever $x \in U^{\prime \prime}$, then $F_{a(1)}(x) \cap V_{a(1)}\left(\phi^{0}\right) \neq 0$, and Hence $f_{a(1)}(x)=$ lub $\left\{y_{a(1)} \mid y_{a(1)} \in F_{a(1)}(x)\right\}$ is greater than $y_{a(1)}-\phi$.

Let $W=\left\{y_{a(1)} \mid y_{a(1)}<y_{a(1)}^{0}+\phi\right\}$. Then $W$ is open and contains $F_{a(1)}\left(x^{0}\right)$ so that (2) in the proof of Theorem 1 implies the existence of an open set $U^{\prime}$ containing $x^{0}$ such that $F(x)$ is contained in $W$ for all $x \in U^{\prime}$, i.e., $x \in U^{\prime}$ implies lub $\left\{y_{a(1)} \mid y_{a(1)} \in F_{a(1)}(x)\right\}<y_{a(1)}^{0}+\phi$.

Now $x \in U^{\prime} \cap U^{\prime \prime}$ implies that $y_{a(1)}^{0}-\phi<y_{a(1)}=f_{a(1)}(x)<y_{a(1)}^{0}+\phi$, and hence $f_{a(1)}$ is continuous.

Let $f\left(x^{0}\right)=y^{0}$. Assume that $f$ is not continuous at $x^{0}$. Then there exists an open set $W$ containing $y^{0}$, a directed set $D$, and a sequence $\left\{x_{d}\right\} \rightarrow x^{0}$ such that $d \in D$ implies that $f\left(x_{d}\right) \notin W$. The set $T-W$ is closed and hence compact. The sequence $\left\{f\left(x_{d}\right)\right\}$ determines a net $\phi$ on $D$ to $T-W$ defined by $\phi(d)=f\left(x_{d}\right)$ and hence [9, Theorem 24] there exists a subnet $(E, \theta)$ with a limit $\bar{y}$ in $T-W$. Let $k$ be the function on $E$ to $D$ satisfying the definition of subnet. Since $\phi(d)=f\left(x_{d}\right)$, $\theta(e)=\phi k(e)=f\left(x_{k(e)}\right)$. If $V$ is an open set containing $\bar{y}$, then there exists $e(V) \in E$ such that $e * e(V)$ implies that $\theta(e) \in V$. If $U$ is an open set containing $x^{0}$, then there exists $d^{\prime}$ such that $d * d^{\prime}$ implies that $x_{d} \in U$. If $d^{\prime} \in D$ then there exists $e^{\prime}$ such that $e * e^{\prime}$ implies that $k(e) * d^{\prime}$. Therefore $e * e^{\prime}$ implies that $x_{k(e)} \in U$. Then $\left\{x_{k(e)}\right\} \rightarrow x^{0}$ and $\left\{f_{a(1)}\left(x_{k(e)}\right)\right\} \rightarrow \bar{y}_{a(1)}$. It was shown that $f_{a(1)}$ is continuous, hence $f_{a(1)}\left(x^{0}\right)=\bar{y}_{a(1)}$. But $f_{a(1)}\left(x^{0}\right)=y_{a(1)}^{0}$, therefore $\bar{y}_{a(1)}=y_{a(1)}^{0}$. Then the hypothesis that $F$ is an apex function implies that $\bar{y}=y^{0}$, which is an element of $W$. But $\bar{y} \in T-W$. This contradiction implies that our assumption was false and $f$ is continuous. This trace $f$ is a continuous single-valued function on a Tychonoff cube to itself and hence Lemma 1 implies that $F$ has a fixed point $x_{0}$. The proof that $h^{-1}\left(x_{0}\right)$ is a fixed point of $G$ is a reiteration of the last statement in the proof of Theorem 2.

\section{BIBLIOGRAPHY}

1. W. L. Strother, Continuous multi-valued functions, Trans. Amer. Math. Soc. (to appear).

2. L. Ratner, Multivalued transformations, University of California, 1949.

3. A. D. Wallace, A fixed point theorem for trees, Bull. Amer. Math. Soc. vol. 47 (1941).

4. S. Eilenberg and D. Montgomery, Fixed point theorems for multi-valued transformations, Amer. J. Math. vol. 68 (1946) pp. 214-222.

5. S. Kakutani, $A$ generalization of Brouwer's fixed point theorem, Duke Math. J. vol. 8 (1941) pp. 457-459. 
6. S. Banach and S. Mazur, Uber mehrdeutige stetige Abbildungen, Studia Math. vol. 6 (1936) pp. 174-178.

7. Ernest Michael, Topologies on spaces of subsets, Trans. Amer. Math. Soc. vol. 71 (1951) pp. 152-182.

8. P. Alexandroff and H. Hopf, Topologie, Berlin, 1935. 283.

9. J. L. Kelley, Convergence in topology, Duke Math. J. vol. 17 (1950) pp. 277-

University of Miami

\section{ERRATA, VOLUME 3}

C. W. Curtis, $A$ note on noncommutative polynomials.

p. 965, line 10 from the bottom. Add to condition (b): "where $T(r) \neq 0$ if $r \neq 0 . "$

\section{ERRATA, VOLUME 4}

W. R. Mann, Mean value methods in iteration.

p. 507, Display (2) should include the following:

$$
\lim _{i \rightarrow \infty} a_{i j}=0 \quad \text { for all } j \text {. }
$$

E. Michael, $A$ note on paracompact spaces.

p. 835, diagram near the top of the page. For "covering" read "open covering" (twice), and for "refinement" read "open refinement" (twice). 